\title{
Philosophiques
}

\section{Achille Mbembe, Brutalisme, Paris, La Découverte, 2020, 246 pages}

\section{Thibault Tranchant}

Volume 47, numéro 2, automne 2020

URI : https://id.erudit.org/iderudit/1075139ar

DOI : https://doi.org/10.7202/1075139ar

Aller au sommaire du numéro

Éditeur(s)

Société de philosophie du Québec

ISSN

0316-2923 (imprimé)

1492-1391 (numérique)

Découvrir la revue

Citer ce compte rendu

Tranchant, T. (2020). Compte rendu de [Achille Mbembe, Brutalisme, Paris, La Découverte, 2020, 246 pages]. Philosophiques, 47(2), 508-512.

https://doi.org/10.7202/1075139ar d'utilisation que vous pouvez consulter en ligne.

https://apropos.erudit.org/fr/usagers/politique-dutilisation/ 
Marx chez Althusser, et des concepts d'histoire et de science chez Althusser et Foucault font toutefois de ce livre un traité d'épistémologie et de matérialisme davantage que de philosophie féministe. Plutôt que d'établir des liens tout au long du texte entre ces auteurs français et Butler, Benoit n'aborde directement la théorie féministe qu'au premier et dernier chapitre, de sorte qu'en somme ce livre plaira avant tout à celles et ceux qui s'intéressent à Althusser et à l'épistémologie marxiste et, de façon secondaire seulement, aux chercheuses en théorie féministe.

CAPUCINE MERCIER

University of New Mexico

Achille Mbembe, Brutalisme, Paris, La Découverte, 2020, 246 pages.

La Grand Theory sociologique et la philosophie de l'histoire ont été marquées dans les années 1980 et 1990 par l'emprunt du concept architectural de «postmodernité». En réaction au formalisme et au rationalisme modernistes, des architectes tels que Robert Venturi ou Michael Graves voulurent dans les années 1960 réhabiliter l'histoire et la culture vernaculaire dans l'ordre architectural. Il en résulta une certaine forme d'hybridation et d'éclectisme esthétiques, voire une «vitalité bordélique ${ }^{1}$ » (messy vitality), neutralisant les référentiels progressistes du modernisme. Des sociologues et des philosophes comme Fredric Jameson, Jean-François Lyotard ou Michel Freitag s'inspirèrent des théories architecturales postmodernistes afin de forger un concept du temps présent. Il fut l'objet de définitions diverses, voire contradictoires. On proposa même de voir dans la "postmodernité» une "hypermodernité ", c'est-à-dire non pas une sortie de la modernité, mais l'accentuation de ses tendances internes (Charles et Lipovetsky).

Le dernier essai d'Achille Mbembe, Brutalisme, réitère cet emprunt conceptuel à l'architecture. "J'emprunte le concept de brutalisme à la pensée architecturale ", écrit l'auteur dès la première ligne de son essai (p. 7). Mais, cette fois-ci, il ne s'agit plus de s'interroger sur la nature d'un processus transitoire, mais bien de décrire les résultats d'un accomplissement. Le brutalisme ayant été un mouvement architectural moderniste, cet emprunt suggère que la "brutalité » est moins le terme d'une éventuelle postmodernité que celui, pathologique, de la modernité elle-même; elle est en ce sens un hypermodernisme. La Cité radieuse de Le Corbusier est parfois citée comme un exemple d'architecture brutaliste: béton apparent, répétition d'éléments simples tels que des fenêtres et des pilotis, gigantisme et utopisme. Le brutalisme architectural agence des matériaux bruts, en particulier le béton, le

1. Robert Venturi, Complexity and Contradiction in Architecture, New York, The Museum of Modern Art, 1966, p. 16. 
verre et le fer, dans une forme esthétique fonctionnaliste et rationaliste. Pour Mbembe, notre époque est "brutale »; elle est à l'image de ces bâtiments qui, tel le Boston City Hall, bouleversent les territoires et paraissent écraser l'imagination sous des tonnes de béton.

Le «brutalisme» est une «domination universelle» rendue possible par «l'étroite imbrication de plusieurs figures de la raison: la raison économique et instrumentale, la raison électronique et digitale et la raison neurologique et biologique» (p. 23). Il repose sur la digitalisation des rapports sociaux et sur leur croissante prise en charge computationnelle, inséparables du développement du capitalisme (p. 64-66). La numérisation est le fondement technique de la brutalisation, elle est « la mâchoire transcendantale qui dessine désormais la carte de notre monde»(p. 65). Mbembe confère une signification ontologique à la «brutalité»: elle fracture les formes et les recompose artificiellement. Comme l'architecte brutaliste qui extrait des entrailles de la Terre la matière brute pour la réagencer, la raison brutale est engagée dans «une vaste entreprise d'occupation de territoires, d'emprise sur les corps et les imaginaires, de désassemblage, de déliaison et de démolition» (p. 14). Il en résulte un anéantissement des dichotomies classiques par lesquelles nous avons appréhendé l'être jusqu'à maintenant: «forme/matière, matière/matériau, matériel/immatériel, naturel/artificiel et fin/moyen » sont pulvérisés par la raison brutale, occupée à reconfigurer l'expérience selon ses propres critères d'efficacité. Aussi le fait majeur de notre temps serait-il l'indistinction du naturel et de l'artificiel moyennant l'arraisonnement technique de la nature, voire sa production ontologique par les techniques: «Le $\mathrm{XxI}^{\mathrm{e}}$ siècle [est] la route vers ce monde de la nature fabriquée et de l'être fabricable [...]. La technologie est finalement parvenue à s'ériger en destinée ontologique de l'ensemble du vivant» (p. 36). Dans ce bouleversement, la société est aussi emportée et remplacée par « un nanomonde» (p. 14).

Brutalisme esquisse les différentes facettes de la brutalité, les diverses figures de la «démolition», du «forçage» et du «concassage»(p. 8). L'essai a lui-même quelque chose de «brutal». Il se présente comme un agencement itératif de thèmes autour d'une idée maitresse (la nature et les conséquences de la brutalité). Il s'agit de qualifier notre époque à partir d'une "imagepensée », un "fond duquel se détache une myriade de situations, d'histoires, d'acteurs» ( $p$ 11). L'épistémologie implicite de cet essai repose sur un concept fort d'imagination productrice, puisqu'il s'agit moins de décrire que de donner à voir par une image inspirée de l'architecture. Ainsi pourrait-on lui reprocher des «biais de confirmation ", une sélection unilatérale de certains phénomènes, si nous ne les rapportions pas à ce geste imaginatif. Comme l'écrivait William Blake: «Ce qui est maintenant prouvé a d'abord été purement imaginé2.»

2. Cité dans Castoriadis, Les carrefours du labyrinthe, 2. Domaines de l'homme, Paris, Seuil, coll. «Points essais», p. 570. 
Parmi les différents thèmes qu'Achille Mbembe explore, la sexualité et la mobilité occupent une place importante. Dans le quatrième chapitre, «Virilisme », l'auteur propose une sorte de généalogie critique de la brutalisation du corps, du sexe et de la jouissance. Il prend pour point de départ la «répression» de la sexualité par la chrétienté pour décrire dans un second temps la colonisation comme le lieu d'une libération libidinale et de la constitution d'un "pouvoir orgastique » (p. 107), c'est-à-dire une prise phallique sur les corps en vue de la jouissance du dominant. La colonie vue sous cet angle est pour Mbembe une figure fondatrice de la brutalité, car elle est le lieu où se déploie une entreprise gigantesque de démolition et de reconstitution des formes, de leur perception et de leur expérience, jusqu'à celles relatives au corps et au sexe: "coloniser, c'est brutaliser» (p. 108). Elle est le lieu où se manifeste paradigmatiquement la destruction créatrice du corps et du rapport à soi autour du "phallos» dans sa variante occidentale. Dans une perspective psychanalytique, Mbembe décrit le phallus comme un signifiant-maître qui donne au pouvoir sa forme et sa dynamique. Il surinvestit la virilité et rend possible, pourrait-on dire avec Rancière, une "police ", une mise en ordre du sensible, des représentations et des accès à la sphère publique (p. 116). En découlent pour Mbembe le patriarcat et l'hétéronormativité (p. 120-122). Condition métapsychologique de la brutalité, Mbembe soutient cependant que le phallus est devenu l'objet de sa violence aveugle. Après avoir brutalisé le monde et cherché à détruire les limites externes à sa jouissance, il se brutalise désormais lui-même, ouvrant ainsi sur sa contestation; sa brutalisation "préfigure peut-être sa prochaine mise en minorité» (p. 124). C'est la raison pour laquelle nous serions rentrés dans «l'ère $[. .$.$] du trafic des signifiants sexuels» (p. 122) et de l'indistinc-$ tion performative de la dichotomie entre l'homme et la femme, "propulsée par les transformations technologiques en cours» (p. 127). Cela signifie-t-il que la contestation du patriarcat participe toujours de la logique qui l'a rendu possible et qu'elle n'est, en ce sens, qu'une ruse de la raison brutale? Il est difficile de cerner les expériences empiriques auxquelles Mbembe renvoie dans ces derniers développements spéculatifs sur l'autobrutalisation du phallus, mais il est surtout malaisé de cerner la signification normative qu'il lui confère. S'agit-il d'une libération ouvrant sur une "plus grande égalité des genres» (p. 129) ou bien de la condition d'une forme nouvelle d'arraisonnement de l'intime par la technique? Mbembe hésite.

La mobilité est un autre thème majeur traversant l'ensemble de Brutalisme. Mbembe repart de l'argumentaire schmittien dans le Nomos de la Terre. Il rappelle comment Schmitt ancrait l'ordre juridique sur une "prise» première de territoire et comment le droit des gens européen évolua dans le cadre de la première mondialisation coloniale lors des "Grandes Découvertes» (p. 59-62). Cette forme élémentaire de brutalisation du sol interpelle Mbembe, car elle lui fournit le contexte à partir duquel penser la transformation des frontières et la brutalisation du fait migratoire. Nous 
assisterions à de nouvelles formes de "prises", de "ponctions» et de "captations ", lesquelles ne concerneraient plus seulement les territoires, mais les individus par-delà les frontières. Nous sommes passés de la frontière à la "frontiérisation », c'est-à-dire à l'autonomisation et à la dissémination de la logique frontalière par-delà la frontière elle-même (p. 66-71). Il ne s'agit plus de surveiller la limite de son territoire, mais de traquer par-delà sa limite ceux qui voudraient la franchir. La marque de la souveraineté n'est plus l'exception, c'est la chasse à l'homme, soutient-il en s'inspirant de Grégoire Chamayou (p. 70). La brutalisation de la frontière produit ainsi une transformation profonde de l'expérience migratoire. Alors que nous partageons une "condition commune de passants» (p. 68), la frontiérisation de la planète rend possible la fragmentation des corps selon des classifications que le projet cosmopolitique voulait abolir: la race, la nation et le sexe (p. 131). Régime brutal du corps, le "corps-frontière» n'est pas seulement celui de l'étranger, mais aussi celui du citoyen sur lequel s'exerce la brutalité d'État (p. 141). Mbembe considère ainsi que la mobilité sera l'un des enjeux majeurs du Xxie siècle. Elle met en jeu le maintien ou la disparition de l'ordre juridico-territorial moderne, mais surtout le statut politique et économique du Nord à l'échelle globale, lequel, soucieux de maintenir son hégémonie dans un contexte démographique qui lui est défavorable, intensifie sa frontiérisation. À la différence de ses réflexions sur la sexualité et le genre, Mbembe tire ici des conclusions relativement claires, bien qu'elles n'ouvrent sur aucune prescription programmatique: il convient «d'inventer d'autres façons d'habiter la planète [...] en imaginant des formes politiques et étatiques et des modalités d'appartenance toujours plus flexibles, pliables et mobiles» (p. 171). La frontiérisation appelle une défrontalisation, mais n'est-ce pas là aussi une brutalisation de la frontière?

L'un des axes les plus stimulants traversant Brutalisme est l'idée selon laquelle la brutalisation du monde implique le «signe africain» (p. 25). Renouvelant une thèse développée par Aimé Césaire dans son Discours sur le colonialisme et par Hannah Arendt dans Les origines du totalitarisme, Mbembe soutient que la brutalisation est un «devenir-nègre ${ }^{3}$ du monde (p. 11). Césaire et Arendt soutenaient que les savoirs et les techniques de pouvoir ayant structuré l'ordre colonial avaient rendu possible l'ordre totalitaire. Mbembe s'empare de ce schème interprétatif et affirme que l'expérience radicale de dépossession et de réification vécue par le "nègre» (concept employé par Mbembe pour définir le sujet ravagé par la brutalité coloniale) est amenée à devenir une condition universelle: l'Afrique est «le laboratoire de mutations d'ordre planétaire» (p. 13). Mais c'est précisément parce que l'Afrique a traversé la brutalité coloniale qu'elle offre aujourd'hui

3. Dans la recension, le mot $\mathrm{n}^{* * *}$ apparaît dans son intégralité toujours dans le cadre de citations extraites du livre recensé, conformément au souhait que l'auteur, Achille Mbembe, a lui-même exprimé après que nous l'ayons consulté à ce sujet. Note du directeur. 
les ressources afin de réparer le «tissu du vivant» (p. 15). «L'Afrique aura paradoxalement, par-delà la blessure, représenté cette réserve de puissance, ou encore cette puissance en réserve, la seule capable de rapatrier l'humain non sur la Terre, mais dans le Cosmos » (p. 24). Au «devenir-nègre» répond le «devenir-africain» (p. 13), c'est en Afrique que «les opportunités de métastases créatrices sont les plus mûres» (p. 24-25).

Il n'est pas impossible qu'Achille Mbembe invente a posteriori une certaine tradition africaine qu'il constitue en opposition à une métaphysique occidentale de la substance (p. 101). On trouve en effet tout au long de Brutalisme des descriptions englobantes de la pensée africaine que l'on accepterait difficilement pour d'autres aires géographiques et qui ne sont, d'ailleurs, pas sans rappeler une forme dépassée d'ethnophilosophie héritée de Placide Tempels que les philosophes africains cherchent aujourd'hui à conjurer ${ }^{4}$. Mbembe soutient par exemple que les "pensées africaines antiques" nous permettent d'opérer une salutaire critique de l'identité en la situant dans le devenir et «le tissu de relations dont chacun était la somme vivante » (p. 99). Mais de quelle Afrique parlons-nous exactement? Et dans quelle mesure une telle métaphysique du devenir est-elle susceptible de se traduire en une politique émancipatrice? Le passage de l'ontologie à la politique, en effet, n'a rien de nécessaire. Un texte comme la Charte de Kurukan Fuga, que l'on désigne parfois comme une voie proprement africaine vers les droits de l'homme, ne nous montre-t-il pas au contraire une organisation statutaire et hiérarchique de la société fort peu compatible avec l'ontologie "fluide" qui est censée la fonder ? Malgré cette difficulté, Mbembe suggère, c'est une des voies prometteuses de cet ouvrage, que l'Afrique peut nous apprendre à «réparer». En ayant envisagé l'être comme un tissu en devenir, les pensées antiques d'Afrique nous offriraient les ressources afin d'apprendre, par-delà la brutalité, à restaurer notre lien au vivant, "à recomposer la relation" (p. 54). Mbembe laisse toutefois en suspens la question de la traduction politique d'une telle écologie réparatrice et de l'institution d'une «démocratie des vivants ».

THIBAULT TRANCHANT

Cégep Édouard-Montpetit

4. Pour une mise au point sur ce débat, cf. Séverine Kodjo-Grandvaux, Philosophies africaines, Paris, Présence Africaine Éditions, coll. "La philosophie en toutes lettres », 2013. 